\title{
Un programa de formación continua con profesores de ciencias en el contexto de la relación Museo-Escuela
}

Recibido: 05-12-2010 | Aceptado: 30-06-2011

A continuing education program to science teachers

in the context of museum-school relationship

Carlos Soto Lombana*

Fanny Angulo Delgado**

René Rickenmann***

Resumen: En este artículo se presentan los resultados deun programa deformación continuacon ocho profesores de ciencias naturales de educación básica y media, que integró el trabajo de dos museos de ciencia de la ciudad de Medellín, Colombia, como herramienta pedagógica y didáctica. Los resultados del programa de formación permiten apreciar el gran potencial de los museos como recurso educativo y didáctico para la enseñanza de las ciencias naturales. Como principal conclusión se plantea que el diseño de las Unidades Didácticas como parte de un ciclo de aprendizaje, es lo que establece la diferencia entre la acción pedagógica del docente en relación con la visita en el contexto formal del que realiza un colectivo escolar a un museo en el contexto informal.
Palabras clave: Formación de profesores de ciencias, Educación en Museos, Unidad Didáctica

\begin{abstract}
This article presents the results of an ongoing training program with eight teachers of natural sciences of primary and secondary education, which integrated the work of two science museums in Medellin, Colombia, as a pedagogical and didactic strategy. The results of the training program show the great potential of museums as educational and training resource for teaching the natural sciences. The main conclusion suggests that the design of teaching units as part of a learning cycle is what makes the difference between the teacher's pedagogical action in relation to the formal visit in the context of performing a school staff to a museum in the informal context.
\end{abstract}

Keywords: Science Teacher Education, Education in Museums, Teaching Unit.

\footnotetext{
* ** Universidad de Antioquia - Colombia. Grupo GECEM. Calle 67 \# 53 - 108. Bloque 9. Oficina 329. Medellín. Tel. (00 $574) 21957$ 27. Correo electrónico: gecem.udea@gmail.com

*** Université de Genève - Suiza. Grupo SED-DEAC. Faculté de Psychologie et des Sciences de l'Éducation. 40, boulevard du Pont d'Arve. CH-1205 Genève. Tel. (00 41 3) 799843. Rene.Rickenmann@unige.ch
} 


\section{La vinculación de los profesores (en formación inicial y continua) con los museos}

Que los docentes son una meta de mercadeo de los museos, es algo que no da lugar a duda. King (2007) al respecto comenta:

Las encuestas siguen informando que la mayoría de los profesores influyen en la decisión de una escuela para usar los recursos del museo. Los museos se ven obligados a ver a los profesores como mercado objetivo clave que debe ser cultivado y cortejado a fin de mantener los actuales niveles de visitas de los grupos escolares (King, 2007: 78)

Más allá de las implicaciones en el orden comercial de lo que significa mantener un segmento bien definido y con una estrategia de mercadeo, es importante reconocer que los docentes juegan un papel estratégico a la hora de fortalecer la relación museo-escuela.

La literatura internacional da cuenta de un grupo importante de trabajos, tanto en el plano de la indagación de las opiniones y percepciones de los docentes con respecto a los museos, el patrimonio, las colecciones, y su posibilidad y potencialidad como recurso para la enseñanza (por ejemplo Anderson, Kisel y Storksdieck, 2006); así como en el plano del diseño, ejecución y valoración de cursos o espacios para la capacitación y la formación de los profesores en el contexto de la relación museo-escuela (por ejemplo Leinhardt y Gregg, 2002).

Entre el primer grupo de interés, se encuentran trabajos que centran su atención en los aspectos museológicos y museográficos. Por ejemplo, Ana $M^{a}$ Wamba y Roque Jiménez (2005), usan un cuestionario para estudiar las percepciones que tienen sobre el patrimonio los docentes de educación primaria y secundaria y los gestores o divulgadores del patrimonio en ciudades como Sevilla, Cádiz y Huelva. El cuestionario explora la definición de patrimonio, su enseñanza y/o difusión y los procesos de identificación personal con dicho patrimonio.

En un segundo grupo de trabajos, se indaga por las visitas y/o viajes de campo de grupos escolares a sitios de importancia histórica y cultural, a manera de estudio de visitantes. Anderson y Zhang (2003) en un estudio realizado en Canadá, se preguntan por los factores que influyen en las decisiones de los docentes sobre la planeación, programación y realización de las visitas de campo. Entre los factores identificados se destacan la relación con los contenidos curriculares, la relevancia histórica y cultural del sitio, el acceso y logística, así como las visitas y las experiencias previas de los docentes.

Anderson, Kisel y Storksdieck (2006) abordan las problemáticas comunes que enfrentan profesores de tres países diferentes (Estados Unidos, Canadá y Alemania), a la hora de realizar una visita con sus estudiantes, así como las sugerencias y propuestas para mejorar la relación entre los museos y las escuelas. Dentro de los problemas detectados, sobresalen las dificultades propias de relacionar dos sistemas educativos con filosofías y propósitos distintos (formal e informal) y entre los más acuciantes, se señalan: la comunicación y cooperación, la dicotomía entre la logístico y lo pedagógico, el acceso y uso de los materiales y la formación de los docentes en el uso de recursos no convencionales.

Los dos trabajos antes reseñados tienen un carácter general, en cuanto se trata de estudios de visitantes y abordan aspectos de las visitas con población escolar; no 
Un programa de formación continua con profesores de ciencias en el contexto de la relación Museo-Escuela Carlos Soto Lombana | Fanny Angulo Delgado | René Rickenmann

obstante, sus objetivos no están dirigidos a indagar por las reflexiones pedagógicas de los profesores sobre el uso del museo como recurso didáctico. En este sentido, el trabajo de Aguilar el at. (2005) realizado en España, estudia las expectativas y vínculos del profesorado que visita el Centro Interactivo Principia, así como sus opiniones y valoraciones sobre la oferta educativa de dicho centro para los grupos escolares.

En la misma línea de la utilización del museo como recurso didáctico, el organismo estatal del Reino Unido MLA (Museums, Libraries and Archives Council), realizó en el 2008 una encuesta con 121 maestros para averiguar cómo las escuelas estaban utilizando los museos y archivos de Londres y cómo estos espacios podían mejorar sus servicios de acuerdo con las necesidades de los docentes.

Esta misma entidad gestionó durante el periodo 2003-2007, varios estudios para evaluar el impacto de diversos programas aplicados en los museos de la ciudad de Londres, sobre el aprendizaje de sus visitantes. Haciendo uso de la técnica de grupos focales con docentes que visitaron los museos, Johnsson (2003) estudió las relaciones que se establecen entre estudiantes y, estudiantes y profesores en el contexto de la visita, el desarrollo de habilidades comunicativas y de pensamiento, las dinámicas de la clase y la motivación por el aprendizaje. Este reporte proporciona algunas recomendaciones para mejorar la relación museo-escuela, de tal manera que los museos contribuyan con el aprendizaje formal.

Lemerise y Matias (2001) identificaron las percepciones de los docentes sobre la función educativa de los museos, sus intereses, necesidades y expectativas frente a estos espacios, sus experiencia de visitas con grupos escolares, los criterios utilizados para juzgar el éxito de una visita o un proyecto en el museo, así como sus opiniones sobre la cuestión general de lo que significa la asociación entre los museos y las escuelas.

Desde los años ochenta ha existido la pregunta por la oferta de cursos y espacios ofrecidos por los museos a los profesores de las escuelas formales. A modo de ejemplo, Gooding (1985) y Heywood (1999) compararon las ofertas de los museos del Reino Unido y de Estados Unidos para la formación de docentes en la utilización de los recursos del museo en las clases. Estos estudios permiten reflexionar sobre las necesidades que tienen los docentes y que podrían ser abordadas por los museos. A este respecto, Peterman (1997) considera que toda acción didáctica que el docente desarrolla, está mediada por sus posiciones sobre el aprendizaje y la enseñanza.

A partir del modelo de las situaciones pedagógicas en el museo propuesto por el grupo canadiense GREM, Aguirre, Vázquez y Rubio (2005) realizaron una propuesta para el curso "Didáctica de las Ciencias Experimentales" donde alumnos de la especialidad de Audición y Lenguaje y de Educación Musical, diseñaron Unidades Didácticas con los recursos del Museo de las Ciencias de Castilla-La Mancha; la población objetivo eran estudiantes de nivel primario y población con discapacidad.

El equipo de Koshi Dhingra et al. (2001) reportó dos cursos de verano (uno sobre Didáctica de las Ciencias y otro en Geología), desarrollados con 40 profesores de primaria y secundaria. Algunos de los docentes estuvieron involucrados en la enseñanza de los cursos, en la organización de un instituto de verano y otros eventos para educadores en el Museo Americano de Historia Natural en Nueva York, así como en la evaluación de impacto del proyecto en los docentes participantes y sus aulas. 
Otro caso interesante de capacitación a docentes en la relación museo-escuela, fue publicado por Lee Schmitt (2002). En este reporte, la capacitación de docentes se hizo en el contexto del Science Museum of Minnesota, en temas como tiempo geológico, paleontología y evolución, a fin de incorporar estos contenidos en el nuevo currículo del Estado de Minnesota. El curso incluyó un programa con conferencias, talleres en los museos, salidas de campo y una serie de prácticas que familiarizaban a los docentes con las temáticas y con los recursos de los museos; además se contó con acceso al personal científico del museo, al material didáctico y a los diversos profesionales que pueden ayudar a planificar viajes de campo.

En cuanto a la formación inicial de profesores, hay dos casos de proyectos dirigidos a profesores en formación inicial: uno en Estados Unidos (Leinhardt y Gregg, 2002), donde 50 estudiantes de Educación en Ciencias Sociales de la región de Alabama visitaron un museo sobre los Derechos Civiles. Más allá de los contenidos de tipo histórico, se buscaba involucrar a los futuros docentes (en su mayoría afro descendientes) en una conexión social y afectiva con el conocimiento de su localidad y de esta manera motivar estos contenidos en sus prácticas educativas.

El segundo proyecto fue desarrollado en Canadá por Anderson, Lawson y MayerSmith (2006), quienes investigaron el impacto de tres semanas de prácticas pedagógicas en un acuario, sobre las percepciones epistemológicas y pedagógicas de un grupo de estudiantes de Educación en Biología. En esta experiencia se analizaron 10 aspectos (visión amplia sobre la educación, imagen de la enseñanza, comprensión de la teoría del constructivismo, competencias de enseñanza, flexibilidad en las técnicas peda- gógicas, trabajo colaborativo, autoconfianza como docentes, trabajo independiente de los estudiantes, papel de la interactividad en el aprendizaje de las ciencias y preparación de la visita), aspectos que emergen cuando se incluye la reflexión sobre el aprendizaje en entornos informales.

En el contexto Latinoamericano, específicamente en los espacios de socialización y encuentro de los miembros de la Red-POP en Brasil, se ha dado un importante desarrollo de propuestas en la relación museo-escuela y algunos trabajos sobre formación docente. Por ejemplo, en 1997 el Departamento de Educación del Museo de Astronomía y Ciencias Afines de la Universidad Federal Fluminense, realizó un curso con 73 docentes del nivel primario: a partir de la exploración del significado de un objeto del museo, se le pidió a los docentes reflexionar sobre su posible aplicación en el aprendizaje de las ciencias (Álvares, 1999).

Por su parte Sepúlveda (1999), del Museo de la Vida en Rio de Janeiro, realizó su tesis doctoral en Museología en el Muséum National d'Histoire Naturelle de Paris, sobre el comportamiento pedagógico de los profesores durante visitas escolares a dos museos científicos. En la publicación que hace el autor en la Red-POP, se valora las acciones pedagógicas que los docentes llevan a cabo en la visita escolar a un museo, el tipo de acción educativa que se privilegia (discusión grupal, trabajo en equipos, trabajo autónomo, etc.); la relación entre el profesor y los objetos, textos o artefactos del museo; las acciones del docente para mediar la relación entre los estudiantes, los objetos y los textos, para organizar el tiempo de la visita, para organizar el viaje; conocer la frecuencia y la naturaleza de las interacciones entre el profesor y los miembros educativos del museo; entre otros. 
Un programa de formación continua con profesores de ciencias en el contexto de la relación Museo-Escuela Carlos Soto Lombana | Fanny Angulo Delgado | René Rickenmann

\section{Trabajos de articulación museo-escuela en el contexto colombiano}

En Colombia, los museos y centros interactivos de ciencia y tecnología son muy recientes. Un primer esfuerzo significativo, aún vigente, fue la conformación del Museo de la Ciencia y el Juego hace 25 años, adscrito a la Universidad Nacional de Colombia (Bogotá). Se trata de una institución museística enfocada al público infantil, centrada en principios y conceptos físicos, que ha logrado popularizar y divulgar la ciencia y la tecnología a través de actividades lúdicas y recreativas. A instancia del Museo de la Ciencia y el Juego, se ha logrado consolidar la Red Liliput, alrededor de la cual gravita una serie de museos de ciencia y tecnología a lo largo y ancho de la geografía nacional (se incluyen algunos museos de Ecuador y Venezuela). El Museo de la Ciencia y el Juego, con el apoyo de la Red-POP (Red de Popularización de la Ciencia y la Tecnología de América Latina) y el Convenio Andrés Bello, ha logrado impulsar seis Escuelas Latinoamericanas de Museología de las Ciencias dirigidas a la formación de recurso humano en el campo de la Educación en los Museos.

En Bogotá, a mediados de la década de los 90s, impulsado por la Asociación Colombiana para el Avance de las Ciencias (ACAC) y otras organizaciones públicas y privadas, se pone en funcionamiento el Centro Interactivo Maloka, dedicado a temas de popularización y divulgación de la ciencia y la tecnología. Por sus orígenes, Maloka no estuvo destinado solamente a constituirse en un espacio de encuentro de la comunidad, sino que se consolidó como un referente educativo alrededor del cual se han desarrollado importantes experiencias de colaboración MuseoEscuela. Dentro de los logros de Maloka está la conformación del Foro Permanente sobre temas de Política Pública en Ciencia y Tecnología, que ha contribuido a la discusión nacional sobre el actual Sistema Nacional de Ciencia y Tecnología.

En Medellín, la dinámica de los centros interactivos es reciente. Como antecedentes se tiene: la conformación de la Sala Interactiva Galileo Galilei, como parte de las colecciones del Museo Universitario de la Universidad de Antioquia a mediados de la década de los 90s y, la creación del Museo Interactivo EPM a comienzos del año 2000. En el 2007 entró a funcionar el Parque Explora, catalogado como centro interactivo de tercera generación, con varias salas interactivas y un acuario, único en su género en Colombia, espacios dispuestos para el disfrute, la investigación y el fortalecimiento de la educación en ciencias.

No obstante, a pesar de la dinámica descrita, en Colombia son escasos los antecedentes de reportes de trabajos colaborativos entre universidades y museos dirigidos a la cualificación del profesorado de ciencias, con el propósito de hacer uso de estos nuevos escenarios como herramienta didáctica de trabajo para fomentar la educación en ciencias y artes. Un antecedente importante lo constituye el programa denominado El Museo un aula más, que tiene su origen en la Cátedra de la Carrera de Bellas Artes de la Universidad Nacional de Colombia en el año de 1986; posteriormente el Ministerio de Educación Nacional lo acogió como proyecto base para la renovación del currículo de la Educación Artística.

Este programa tiene su expresión en Antioquia bajo la denominación de El Museo un aula más en la vida cultural de los estudiantes (González y Velásquez, 1995). Dentro de los aportes del proyecto a la transformación de las prácticas educativas se destacan: 
Los resultados obtenidos en la etapa piloto, permitieron comprobar la validez e importancia del proyecto en la cualificación de los docentes, en la formación integral del estudiantado, en la inmediata aplicación de la propuesta al campo de la educación, en la participación directa de los padres de familia, en la cualificación de los museos $y$ en el restablecimiento de los vínculos entre la educación y la cultura (González \&Velásquez, 1995:4).

El éxito del programa condujo a su implementación en 40 establecimientos educativos del Valle de Aburrá y en 24 Municipio del Departamento de Antioquia. En total se logró la articulación de 21 museos dentro del contexto del programa. Infortunadamente el proyecto no tuvo sostenibilidad y su continuidad no se pudo dar. Gran parte del personal de los museos de la ciudad de Medellín y del Valle de Aburrá desconoce el impacto del esfuerzo, y en la actualidad no existe una política pública o intenciones de las instituciones gubernamentales por revivir el programa.

En el año 2007, en el marco de un proyecto de investigación internacional que ejecutaron los grupos Educación en Ciencias Experimentales y Matemáticas (GECEM) de la Universidad de Antioquia y Semiosis, Educación y Desarrollo (SED) de la Universidad de Ginebra, se logró propiciar una dinámica que involucró algunos museos y centros interactivos de ciencia y tecnología de la ciudad de Medellín, dentro del contexto del proyecto DiCiArte ${ }^{1}$ (Rickenmann, Soto y Angulo, 2007).

Producto del proyecto DiCiArte fue la realización de un programa de formación continua con profesores de educación

1 DiCiArte: Recursos Didácticos Museo-Escuela para la Formación Inicial y Continua del Profesorado de Ciencias. básica y media, en torno a la utilización de los museos como herramienta didáctica y pedagógica. Se buscó estudiar el impacto de los museos en el contexto de las prácticas docentes y la ampliación del referente didáctico de los profesores. A continuación se hace una presentación de la metodología, algunos de los resultados más relevantes y conclusiones de esta experiencia de formación docente.

Las preguntas que orientaron la intervención fueron:

- ¿De qué forma/s adapta el profesor los contenidos científicos del museo, al alumno?

- ¿De qué manera, las actividades en el museo permiten a los estudiantes la elaboración de explicaciones respecto al tema curricular / objeto expuesto?

- ¿Cómo se establece la relación entre el aprendizaje de contenidos conceptuales, con la afectividad, la imaginación, las sensaciones y estímulos que surgen como producto de la contemplación e interacción con los objetos expuestos?

- ¿Cómo influye el museo en la adquisición de nuevos conocimientos, habilidades o actitudes?

\section{Metodología}

El programa de formación continua (curso) se desarrolló en el segundo semestre académico del año 2008; contó con una intensidad de 64 horas distribuidas en 16 encuentros presenciales de cuatro horas cada uno. Se abordaron temas relacionados con tópicos de Educación en Museos (modelo contextual de aprendizaje (Falk y Dierking, 1992), aprendizaje por libre elec- 
Un programa de formación continua con profesores de ciencias en el contexto de la relación Museo-Escuela Carlos Soto Lombana | Fanny Angulo Delgado | René Rickenmann

ción (Falk y Dierking, 2002), modelo pedagógico GREM (Allard y Boucher, 1998), perspectiva constructivista de los museos (Hein, 2005), contenidos museográficos de algunos museos (incluyó la visita al Museo Universitario de la Universidad de Antioquia (Colecciones de Ciencias Naturales y Ser Humano y Sala Interactiva Galileo Galilei), Museo Interactivo EPM, Parque Explora y Museo de Antioquia), así como conocimientos sobre modelos didácticos (Teoría de las Situaciones Didácticas de Brousseau (1996) y diseño de Unidades Didácticas. El tema de las Unidades Didácticas recibió una atención especial, en la medida que representó la estrategia mediante la cual los profesores estructuraron las secuencias didácticas en el contexto del ciclo de Aprendizaje (Jorba y San Martí, 1996).

La visita a los museos de la ciudad incluyó el conocimiento de las colecciones, el guión museográfico, aspectos de orden administrativo y de manera especial los programas y proyectos educativos que poseen estos centros educativos y culturales. Además, el curso contó con expertos que compartieron experiencias en el campo de la divulgación y popularización de la ciencia y la tecnología que complementaron los enfoques educativos de los museos.

Los museos seleccionados para hacer la intervención didáctica fueron: Museo Universitario de la Universidad de Antioquia (Colección Ser Humano y Sala Interactiva Galileo Galilei) y Parque Explora (Sala Abierta con experiencias interactivas de física). Se conformaron cuatro grupos de dos profesores cada uno. Se realizaron video-entrevistas antes y des- pués de la aplicación de la Unidad Didáctica y se filmaron los tres momentos de la intervención: antes, durante y después. A continuación se presentan algunos de los resultados del curso.

\section{Resultados y discusión}

En la Tabla 1 se presentan los principales aspectos que fueron incluidos por los profesores como parte de las actividades preparatorias, de visita y de cierre (antes, durante y después), además del objetivo de la Unidad Didáctica, la Institución Museística, el grado escolar intervenido y el aporte del museo en el contexto del aprendizaje de los estudiantes.

Resulta interesante constatar que los profesores coinciden en afirmar que el museo exhibe los objetos de una manera distinta a como se presentan en la escuela, lo cual les permite tomar ventaja para el refuerzo o contraste del contenido. En consonancia con lo anterior, el papel del museo surge para ellos como espacio de ilustración (experimentación), de refuerzo o de evaluación de los contenidos. En algunas respuestas se destaca la percepción de motivación hacia el aprendizaje, que genera la visita al museo. Esto indica que la relación didáctica establecida por los profesores, sitúa al museo como un instrumento subordinado a los contenidos curriculares a desarrollar, en tanto ofrece las posibilidades de servir como espacio para ilustrar o ver de otras maneras -distintas a las escolares-, el contenido a aprender, así como para favorecer competencias de argumentación y explicación. Con el fin de introducir la discusión de los resultados, a continuación se retoman las preguntas formuladas. 


\begin{tabular}{|c|c|c|c|c|c|c|}
\hline \multirow{5}{*}{ 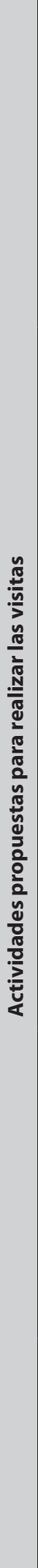 } & 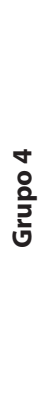 & 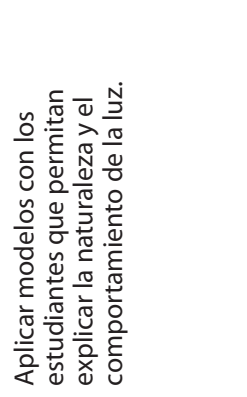 & 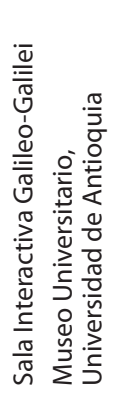 & 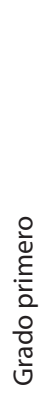 & 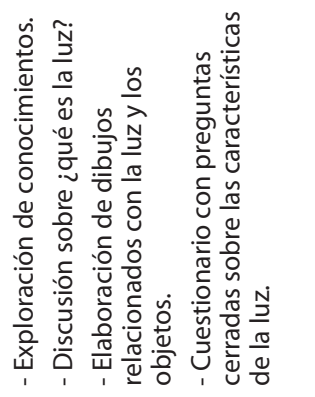 & 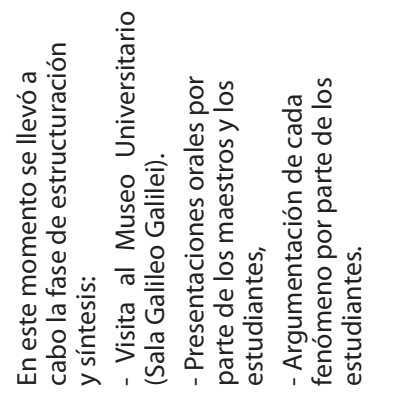 \\
\hline & \begin{tabular}{l}
$m$ \\
$\stackrel{m}{\circ}$ \\
\multirow{2}{2}{}
\end{tabular} & 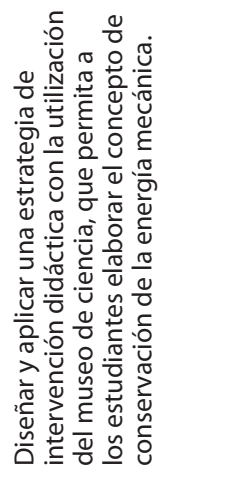 & 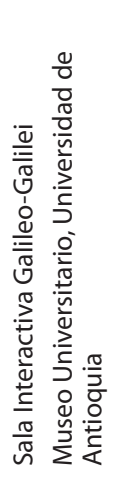 & 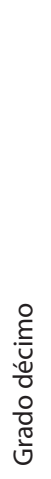 & 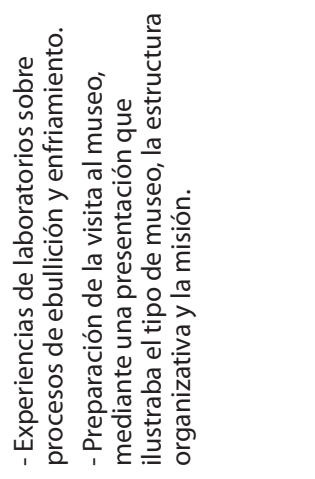 & 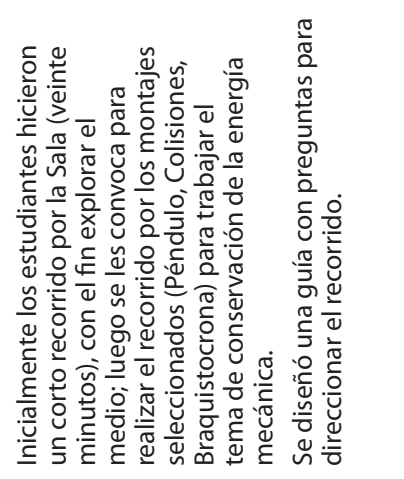 \\
\hline & 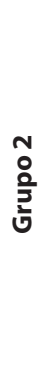 & 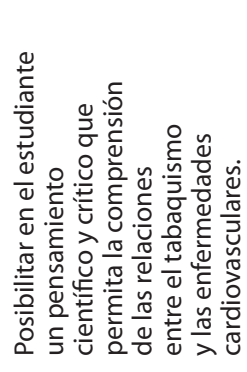 & 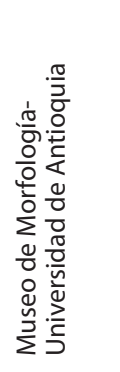 & 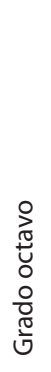 & 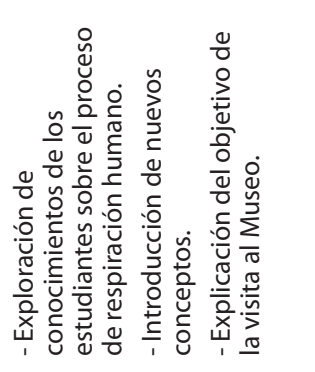 & 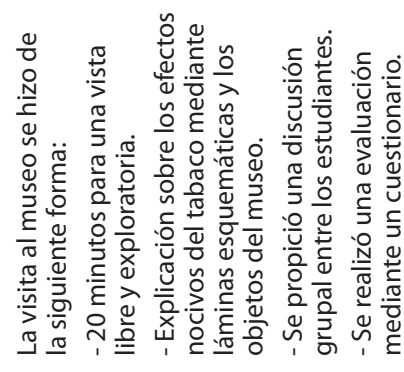 \\
\hline & $\begin{array}{l}\overline{0} \\
\frac{0}{3} \\
\text { 닌 }\end{array}$ & 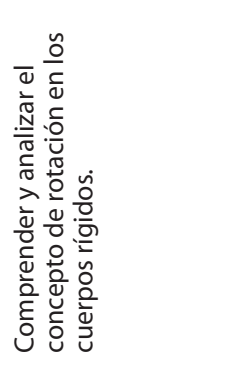 & $\begin{array}{l}\frac{\pi}{0} \\
\frac{0}{2} \\
\frac{1}{x} \\
\frac{0}{0} \\
\frac{0}{0} \\
\frac{0}{0}\end{array}$ & 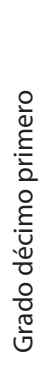 & 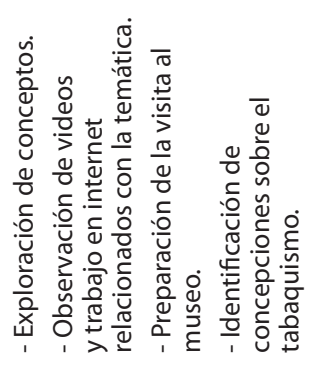 & 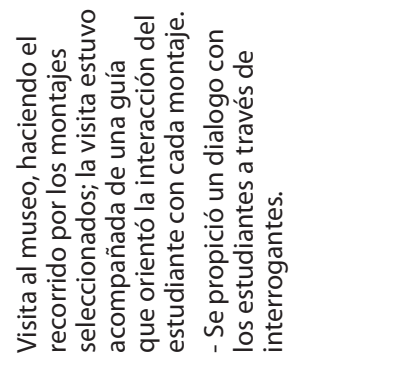 \\
\hline & & 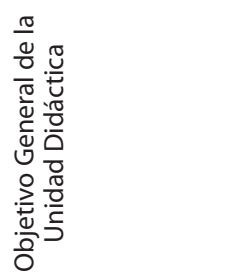 & 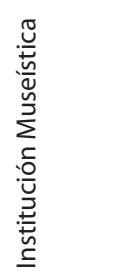 & 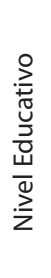 & 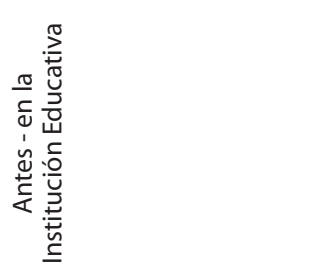 & 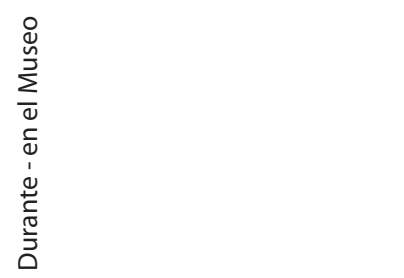 \\
\hline
\end{tabular}




\begin{tabular}{|c|c|c|c|}
\hline \multirow{5}{*}{ 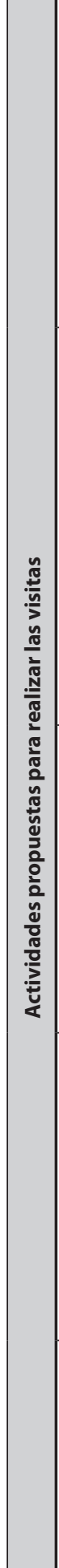 } & $\begin{array}{l}+ \\
\circ \\
\frac{0}{3} \\
50\end{array}$ & 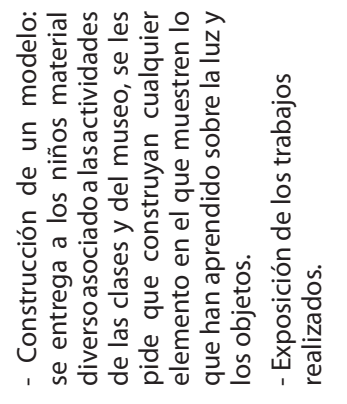 & 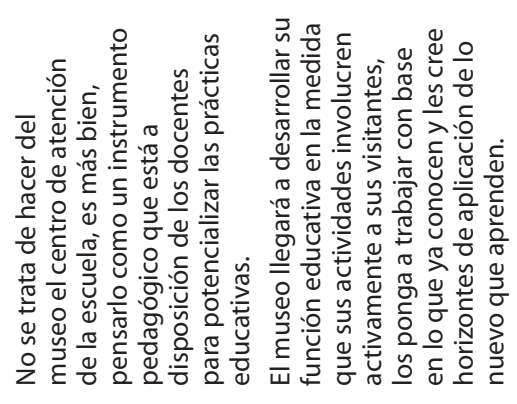 \\
\hline & $\begin{array}{l}\text { mo } \\
\circ \\
\frac{0}{2} \\
5\end{array}$ & 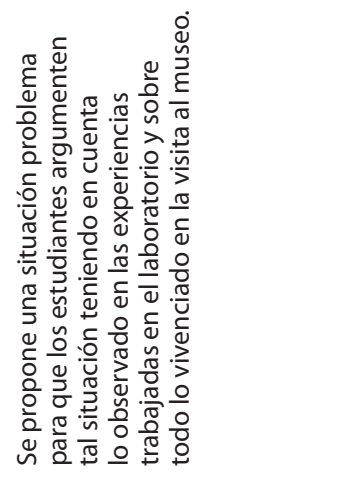 & 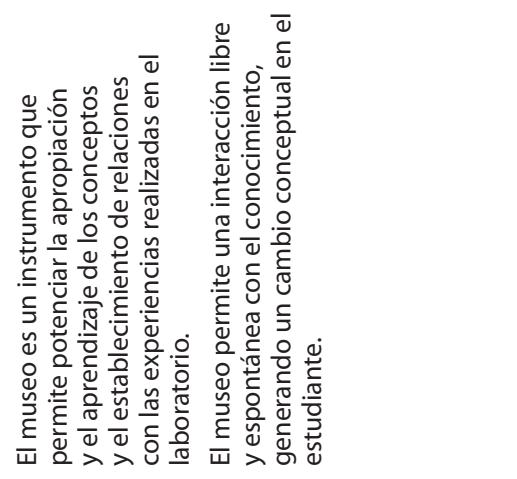 \\
\hline & $\begin{array}{l}\text { No } \\
\text { ํㅗㄴ } \\
\text { ㄴ. }\end{array}$ & 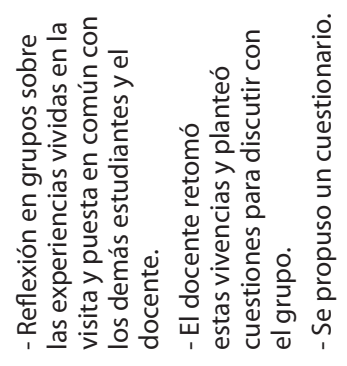 & 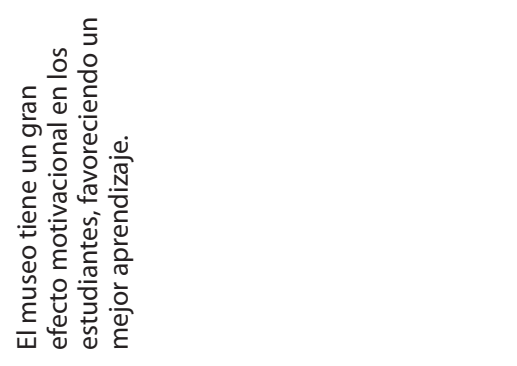 \\
\hline & $\begin{array}{l}\bar{\circ} \\
\frac{0}{2} \\
\frac{5}{0}\end{array}$ & 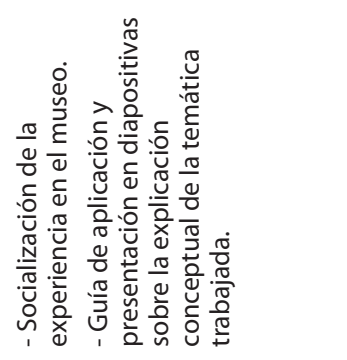 & 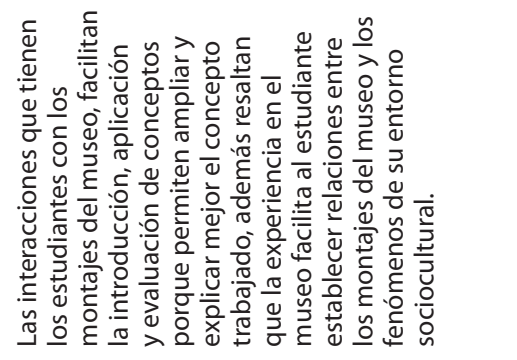 \\
\hline & & 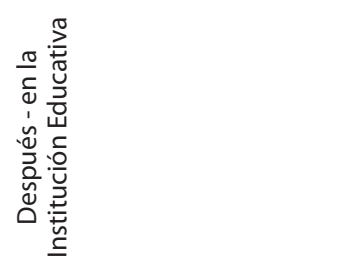 & 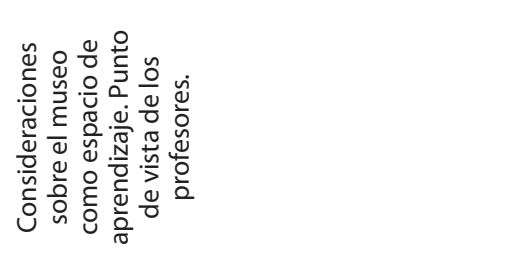 \\
\hline
\end{tabular}

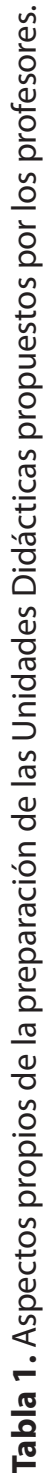


¿De qué forma/s adapta el profesor los contenidos científicos del museo, al alumno?

Esta pregunta se resuelve principalmente con el análisis de las 'Actividades Propuestas' (ver tabla 1) antes, durante y después de la visita. Lo primero a explicitar es que los profesores de los grupos 1, 2 y 4, adoptaron del modelo GREM, la conexión entre la visita en sí (momento durante), con su preparación (momento antes) y con el posterior aprovechamiento de la experiencia (momento después). No obstante, las actividades propuestas no responden necesariamente al modelo didáctico adaptado a la educación museística, armonizado a un aprendizaje inductivo. Si bien los profesores realizan actividades de exploración, en esta etapa las actividades no conducen a que los estudiantes se planteen preguntas que vayan a resolver en el museo, sino que más bien, el profesor recoge información sobre los puntos de partida de los estudiantes respecto al tema y les ayuda a ellos a tomar conciencia de los mismos. Esto puede explicar por qué el museo tiene para ellos un papel de espacio de contrastación del discurso escolar.

En los grupos 2 y 4 , los estudiantes pasan por las exhibiciones y son atendidos por el Educador del Museo (ya sea el guía o el profesor), con actividades dirigidas a la elaboración de explicaciones cercanas a las científicas y distintas a las cotidianas. La capacidad del profesor de adaptar el contenido científico del museo a las características de aprendizaje de sus estudiantes, está determinadas por el tipo de actividades propuestas a los alumnos, que se centran en el diálogo para elaborar la explicación.

El grupo 3 por su parte, parece atender a un modelo inductivo, en cuanto proponen a los estudiantes una guía con preguntas, que empezó a ser planteada en las activida- des del momento 'antes', donde tuvo lugar la exploración e interrogación. El grupo 1 se acercó al modelo inductivo en la etapa donde socializaron la experiencia en el museo y luego presentaron a los estudiantes una guía de aplicación y un grupo de diapositivas sobre la explicación conceptual de la temática trabajada. Esta actividad estuvo acompañada de una serie de problemas muy afines a las situaciones experimentales vistas en el museo, que surtieron el efecto de ejemplificación y contraste con las experiencias.

El enfoque didáctico tiene que ver entonces, con las concepciones que mantienen los profesores sobre cómo creen que aprenden los alumnos, porque están ubicados en el contexto escolar formal, más que en el de la educación museística. Visto así, el aprendizaje de los estudiantes no se establece con la temática del museo, sino con los contenidos curriculares. De esta manera el objeto expuesto en el museo es asimilado al tema curricular, sin que el profesor establezca una distinción entre ambos, en la medida que el profesor concibe al museo como un instrumento para corroborar el texto de saber.

¿De qué manera, las actividades en el museo permitieron al estudiante la elaboración de explicaciones respecto al tema curricular / objeto expuesto?

Los profesores propusieron a sus alumnos actividades en las que se destaca: la explicación del profesor y el diálogo con el alumno, pero más que sobre el objeto expuesto, sobre el contenido curricular. En este sentido, la acción del docente a través de las actividades de enseñanza parece descartar de plano, las posibilidades que ofrecería el museo si las actividades se propusieran desde esta institución y no desde la escuela. 
Un programa de formación continua con profesores de ciencias en el contexto de la relación Museo-Escuela Carlos Soto Lombana | Fanny Angulo Delgado | René Rickenmann

¿Cómo se establece la relación entre el aprendizaje de contenidos conceptuales, con la afectividad, la imaginación, las sensaciones y estímulos que surgen como producto de la contemplación e interacción con los objetos expuestos?

El contraste de la planificación con la entrevista, pone en evidencia en los grupos 1, 3 y 4, que en el discurso oral (entrevista) y en la planificación de la unidad didáctica, los profesores toman conciencia del enorme potencial del museo para favorecer los aspectos sensitivos y afectivos del aprendizaje. Sin embargo, las actividades se centran en los contenidos conceptuales, colocando en un plano implícito estos aspectos, excepto para el grupo 2, que muestra un interés explícito del profesor por indagar en la experiencia afectiva de los estudiantes con la visita.

¿Cómo influye el museo en la adquisición de nuevos conocimientos, habilidades o actitudes?

Esta pregunta se comienza a resolver con las consideraciones que hacen los profesores sobre el museo (ver tabla 1), en cuanto facilita la consolidación de los conceptos (Ej.1.La experiencia en el museo facilita al estudiante establecer relaciones entre los montajes del museo y los fenómenos de su entorno sociocultural; Ej. 2. El museo es un instrumento que permitió potenciar la apropiación y el aprendizaje del concepto y el establecimiento de relaciones con las experiencias realizadas en el laboratorio; Ej. 3. El museo llegará a desarrollar su función educativa en la medida que sus actividades involucren activamente a sus visitantes, los ponga a trabajar con base en lo que ya conocen y les cree horizontes de aplicación de lo nuevo que aprenden) y genera motivación (Ej. El museo tiene un gran efecto motivacional en los estudiantes, favoreciendo un mejor aprendizaje).

\section{Conclusiones}

El programa de formación continua con profesores de ciencias naturales permitió redimensionar el papel educativo de las instituciones museísticas seleccionadas. Las visitas desarrolladas se ubicaron en el contexto de la educación formal, en la medida que se articularon a los lineamientos curriculares, los contenidos y los objetivos de cada nivel educativo seleccionado. En este contexto cada museo se convirtió en lugar o espacio para el aprendizaje, en donde cada profesor desarrolló su labor docente, sin recurrir a la ayuda o concurso de los mediadores de la institución.

Dentro de los aspectos interesantes para resaltar de la experiencia de formación del profesorado en el campo de la relación museo-escuela se pueden mencionar los siguientes:

- Se desprende del seguimiento a las actividades propuestas y desarrolladas por los profesores tanto en la Escuela como en el Museo, como del análisis de las entrevistas, que el curso permitió un cambio de concepción sobre el rol de estos centros culturales como apoyo a la docencia que se desarrolla en el sistema escolar.

- Fue evidente el replanteamiento de las prácticas pedagógicas en el sentido de incorporar la riqueza museográfica de las instituciones museísticas, además de los elementos de tridimensionalidad que caracterizan los montajes y exhibiciones, recursos que no posee la escuela, para la enseñanza y aprendizaje de contenidos escolares.

- Un aspecto en el que vale la pena insistir es el relacionado con la necesidad de la preparación de las visitas al museo, den- 
tro del contexto escolar. En este sentido, el diseño de las Unidades Didácticas como parte de un ciclo de aprendizaje, es lo que marca la diferencia entre la acción pedagógica del docente en relación con la visita en el contexto formal del que realiza un colectivo escolar a un museo en el contexto informal. Es claro que los mediadores que tiene el museo, representados por los guías, no tiene la función y la preparación para abordar esta clase de tareas; no obstante, el profesor con una adecuada fundamentación y experiencia en el trabajo con los museos como recurso educativo, puede complementar esta nueva función del museo: apoyar con sus contenidos y propuestas museográficas el aprendizaje de las ciencias.

- Un programa como el presentado aquí, se constituye en argumento de peso para fomentar la articulación de iniciativas entre las instituciones museísticas y los centros educativos, en la creación de vínculos educativos y pedagógicos que relacione el contenido conceptual de los museos con el contenido curricular del sistema educativo, en un plano de complementariedad que no diluya la misión de cada institución en la otra.

\section{Reconocimientos y agradecimientos}

La presenta investigación fue posible gracias al apoyo y respaldo brindado por las Universidades de Ginebra y Antioquia y de manera especial al Programa de Sostenibilidad de la última Institución que permitió financiar la realización del curso. De igual manera los autores desean agradecer a los ocho profesores que de manera comprometida participaron en el Programa de Formación Docente desarrollado en el marco del Programa de Maestría en Educación de la Universidad de Antioquia.

\section{Bibliografía}

Aguilar, Ma., Blanco, A., Cardenete, S., Durán, C. y Peláez, J. (2005). Valoración del Profesorado de un Centro de Ciencia Interactivo: Principia. Enseñanza de las Ciencias, N. Extra. VII Congreso Internacional sobre Investigación en Didáctica de las Ciencias. Granada, 7 a 10 de septiembre.

Aguirre, C., Vázquez, A. y Rubio, I. (2005). Educación en museos: utilización del museo de las ciencias de castilla-la mancha como recurso no formal y desarrollo de una actividad de juego de roles sobre discapacidad dentro del museo. Enseñanza de las Ciencias, Número Extra. VII Congreso Internacional sobre Investigación en Didáctica de las Ciencias.

Allard, M., Larouche. M.C., Meunier, A. y Thibodeau, P. (1998). Guide de planification et d'évaluation des programes éducatifs. Montreal: Les Éditions Logiques, 239 páginas.

Álvares, M. E. (1999). Uma experiência no museu com um objeto científico. Memorias de la VI Reunión de la Red-POP. 14 al 17 de junio. Río de Janeiro.

Anderson, D., Kisel, J. y Storksdieck, M. (2006). Understanding teachers' perspectives on field trips: Discovering common ground in three countries. Curator 49(3), 365-386.

Anderson, D., Lawson, B. y Mayer-Smith, J. (2006). Investigating the Impact of a Practicum Experience in an Aquarium on Preservice Teachers. Teaching Education 17(4), 341-353

Anderson, D. y Zhang, Z. (2003). Teacher perception of field trip planning and implementation. Visitor Studies Today, 6(3), 6-12.

Boucher, S. \& Allard, M. (1998). Éduquer au Musée: un modèle théorique de pédagogie muséale. Hurtubise, Montreal.

Brousseau, G. (2007). Iniciación al estudio de la Teoría de las Situaciones Didácticas. Trad. Dilma Fregona. Primera edición. Buenos Aires: Libros del Zorzal. 
Un programa de formación continua con profesores de ciencias en el contexto de la relación Museo-Escuela Carlos Soto Lombana | Fanny Angulo Delgado | René Rickenmann

Dhingra, K., Miele, E., MacDonald, M. and Powell, W. (2001). Museum - Collage School: A Collaborative Model for Science Teacher Preparation. In Proceedings Annual Meeting of the American Educational Research Association (Seattle, WA, April 10-14). Documento ERIC: ED454050

Falk, J. and Dierking, L. (2002). Lessons without limit: how free-choice learning is transforming education. Rowman Altamira.

Falk, J. and Dierking, L. (2001). Free-choice science education: how we learn science outside of school. RSM Press.

Falk, J. and Dierking, L. (1992). The museum experience. Washington, DC: Howells House.

González, M. \& Velásquez, P. (1995) Programa: El Museo un aula más en la vida cultural de los estudiantes. Secretaria de Educación y Cultura de Antioquia: Medellín: Material multicopiado.

Heywood, S. (1999). Teaching teachers about museums. Journal Education in Museum, 20, 28-31

Hooper-Greenhill, E. (2007) Museums and Education. London: Routledge

Hooper-Greenhill, E. (1991). Museum and Gallery Education, Leicester, Leicester University Press

Johnsson, E. (2003). Teachers' ideas about learning in museums. [version on line disponibel en: http://www.mlalondon.org.uk/uploads/ documents/HUBteacherideas.pdf]

Jorba, J. y SanMartí, N. (1996). Enseñar, Aprender y Evaluar: Un proceso de Regulación Continua. Propuestas Didácticas para las áreas de Ciencias de la Naturaleza y Matemáticas. Ministerio de Educación y Cultura: Madrid.

King, B. (2007). New Relationships with the Formal Education Sector. In B. Lord (Ed.) The Manual of Museum Learning (77-105) UK: Altamira Press.
Leinhardt, G. and Gregg, M. (2002). Burning Buses, Burning Crosses. Studens Teachers See Civil Rights. In G. Leinhardt, K. Crowley and K. Knutson (Ed.) Learning Conversations in Museums. (139-166). New Jersey: Lawrence Erlbaum

Lemerise, T. and Matias, V. (2001). Volet $3:$ : À travers l'oeil des enseignants et des enseignantes de l'ordre du secondaire». Projet d'enquête sur la relation musées-adolescents. [http://www.unites.uqam.ca/lance/pages/ projets/rapports/Rapportvolet3.pdf]

MLA: Museums, Libraries and Archives, Reino Unido (2008). Participation Research: London teachers' engagement with museums and archives. [http://www.mlalondon.org. uk/uploads/documents/MLA_London, Strategic_Commissioning_Report.doc]

Peterman, F. (1997). Becoming constructivist museum educators. Journal Education in Museum,18, 4-7.

Rickenmann, R. Soto, C. y Angulo, F. (2007). Recursos Didácticos Museo-Escuela para la Formación Inicial y Continua del Profesora de Ciencias. Proyecto de Investigación: Universidad de Antioquia - Universidad de Ginebra.

Schmitt, L. (2002). Helping Teachers Teach: A Learner-Centered Approach to Professional Development. [http://www.astc.org/pubs/ dimensions/2002/nov-dec/teachers.htm]

Wamba, A. y Jiménez, R. (2005). La enseñanza y difusión del patrimonio y la alfabetización científica: Relaciones Ciencia, Tecnología, Sociedad y Patrimonio. Enseñanza de las Ciencias. No. Extra. VII Congreso Internacional de Investigación en Didáctica de las Ciencias.

Xanthoudaki, M. (Editora) (2003). Un Lugar para descubrir: la enseñanza de la ciencia y la tecnología en los museos. Italia: Impaginazione e stampa T\&T Studio. Versión electrónica disponible en: [http:// www.museoscienza.org/smec/pdf_spa/ Libro_espanol_web.pdf] 